\title{
Experimental investigation of dynamic performance of a prototype hybrid tuned mass damper under human excitation
}

\author{
Nima Noormohammadi, Paul Reynolds \\ Department of Civil and Structural Engineering, Vibration Engineering Section, \\ 36/38 Victoria Street, Sheffield, South Yorkshire, United Kingdom, S3 7QB
}

\begin{abstract}
Current sport stadia designs focus mainly on maximizing audience capacity and providing a clear view for all spectators. Hence, incorporation of one or more cantilevered tiers is typical in these designs. However, employing such cantilevered tiers, usually with relatively low damping and natural frequencies, can make grandstands more susceptible to excitation by human activities. This is caused by the coincidence between the activity frequencies (and their lowest three harmonics) and the structural natural frequencies hence raising the possibility of resonant vibration. This can be both a vibration serviceability and a safety issue.

Past solutions to deal with observed or anticipated vibration serviceability problems have been mainly passive methods, such as tuned mass dampers (TMDs). These techniques have exhibited problems such as lack of performance and offtuning caused by human-structure interaction. To address this issue, research is currently underway to investigate the possible application of hybrid TMDs (HTMDs), which are a combination of active and passive control, to improve the vibration serviceability of such structures under human excitation.

The work presented here shows a comparative experimental investigation of a passive TMD and a prototype HTMD applied on a slab strip structure. The most effective control algorithm to enhance the performance of the HTMD and also deal with the off-tuning problem is investigated. The laboratory structure used here is an in-situ cast simply-supported post-tensioned slab strip excited by forces from a range of human activities.
\end{abstract}

Keywords: hybrid tuned mass damper, vibration serviceability, human induced vibration control, genetic algorithm

\section{INTRODUCTION}

Concert arenas and grandstands have the potential to be exposed to a range of human activities, such as jumping and bobbing. These can be a problematic source of vibration in modern stadia as a consequence of growth in material science and structural design techniques leading to slender and lightly damped structures, more frequent use of sports stadia for live music performances and also the changing behavior of spectators who tend to engage in more synchronized activities [1-4].

To fulfill vibration serviceability requirements in civil structures, many types of control systems have been investigated and implemented including passive, active, semi-active and hybrid vibration control.

Passive vibration control is a relatively conventional method to enhance the damping and sometimes stiffness of a structure through application of additional dissipating materials or devices to the structure. The relatively straightforward design and operation without requirement for external power sources make passive control an attractive control methodology [5]. However, lack of full engagement at low levels of vibration [6,7] and problems with off-tuning are some of negative aspects of this type of control [8-10]. In stadia structures, the potential for off-tuning arises due to the variation of structural natural frequency in the presence of human occupants $[11,12]$.

Hybrid control is a combination of passive and active control where the structural response reduction is mainly achieved by energy dissipation through the passive part, whilst the active part enhances its performance. Having smaller actuators and lower power requirements are some of the advantages of employing hybrid control $[7,13]$.

Active and Passive Smart Structures and Integrated Systems 2013, edited by Henry A. Sodano, Proc. of SPIE Vol. 8688, 86880W · (c) 2013 SPIE · CCC code: 0277-786X/13/\$18 doi: $10.1117 / 12.2010656$ 
The work presented here compares the performance of a passive tuned mass damper (TMD) and a prototype hybrid tuned mass damper (HTMD) on a laboratory structure. The control algorithm proposed by the authors in $[14,15]$ is verified experimentally. In addition, a Genetic Algorithm (GA) as a suitable optimization method is introduced to optimize the feedback gains.

\section{MODEL OF THE STRUCTURE}

The laboratory structure used in this study is a simply supported post-tensioned slab strip with length $10.8 \mathrm{~m}$, width $2.0 \mathrm{~m}$ and depth $0.275 \mathrm{~m}$. The total weight of the slab is approximately 15 tonnes [16].

Considering the first bending mode of vibration as a single degree of freedom (SDOF) system, the structure has frequency $f_{s}=4.44 \mathrm{~Hz}$ with modal mass $m_{s}=7150 \mathrm{~kg}$, damping coefficient $c_{s}=1976 \mathrm{Ns} / \mathrm{m}$, stiffness $k_{s}=5,588,071 \mathrm{~N} / \mathrm{m}$ and damping ratio $\xi_{\mathrm{s}}=0.5 \%$. This mode is susceptible to human activities such as walking, iumping and bobbing [17].

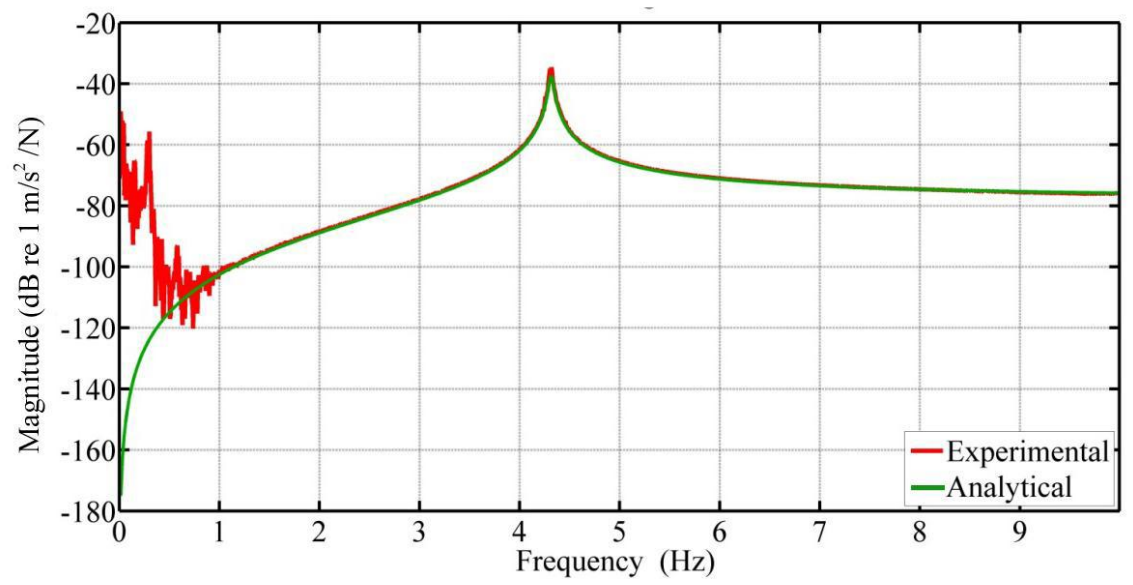

Figure 2-1. FRF magnitude of the uncontrolled structure

The equation of the motion of the uncontrolled structure subjected to external force $\left(F_{e x t}\right)$ is given as

$m_{s} x_{s}(t)+c_{s} x_{s}(t)+k_{s} x_{s}(t)=F_{e x t}(t)$

Converting equation ( 1 ) from time domain to Laplace domain,

$m_{s} s^{2} X_{s}(s)+c_{s} s X_{s}(s)+k_{s} X_{s}(s)=F_{\text {ext }}(s)$

Then,

$\frac{X_{s}(s)}{F_{e x t}(s)}=\frac{1}{m_{s} s^{2}+c_{s} s+k_{s}}$

To achieve the output as acceleration instead of displacement, equation ( 3 ) is multiplied by an $s^{2}$ term, hence the transfer function between an external force (e.g. jumping force) and the output (i.e. structural acceleration) is calculated as

$H_{u n c}=\frac{X_{s}(s) s^{2}}{F_{\text {ext }}(s)}=\frac{s^{2}}{m_{s} s^{2}+c_{s} s+k_{s}}$

where $F_{\text {ext }}(s)$ and $X_{s}(s)$ are the Laplace transforms of the external force input (e.g. human jumping force) and output (i.e. structural acceleration) respectively. Figure 2-1 shows a comparison between the frequency response function (FRF) magnitudes of the analytical model of the structure and the corresponding experimental modal analysis (EMA) result 
below $10 \mathrm{~Hz}$. The higher modes are omitted since the possibility of their significant excitation by human activities is lower [16]. Also, it should be noted that the frequency of the structure in Figure 2-1 is shifted from 4.44 to 4.33 due to the presence of additional mass on the slab during the EMA (which was the locked TMD acting as a passive mass on the structure).

\section{HYBRID TUNED MASS DAMPER}

The HTMD used in this research work comprised of a TMD with an active inertial actuator attached to the passive mass. As was demonstrated in [15], it is possible to replace the actuator dynamic system with just one inertia force (i.e. $F_{I, a c t}$ ), as shown in Figure 3-1. The structural vibration energy is dissipated through the passive part and the active element improves the performance of the system by dealing with the off-tuning problem and reduced performance of passive TMD under low level vibrations. The active element can also be used to augment the damping force of the passive part of the TMD.

As is illustrated in Figure 3-1, the model of the system is a two degree of freedom (DOF) arrangement including the main structure as the first DOF, the passive TMD as the second DOF and an active actuator as the inertia force. $m_{p}, c_{p}$ and $k_{p}$ are the mass, damping and stiffness of the passive part respectively. $x_{p}$ is the displacement of the passive part of the HTMD.

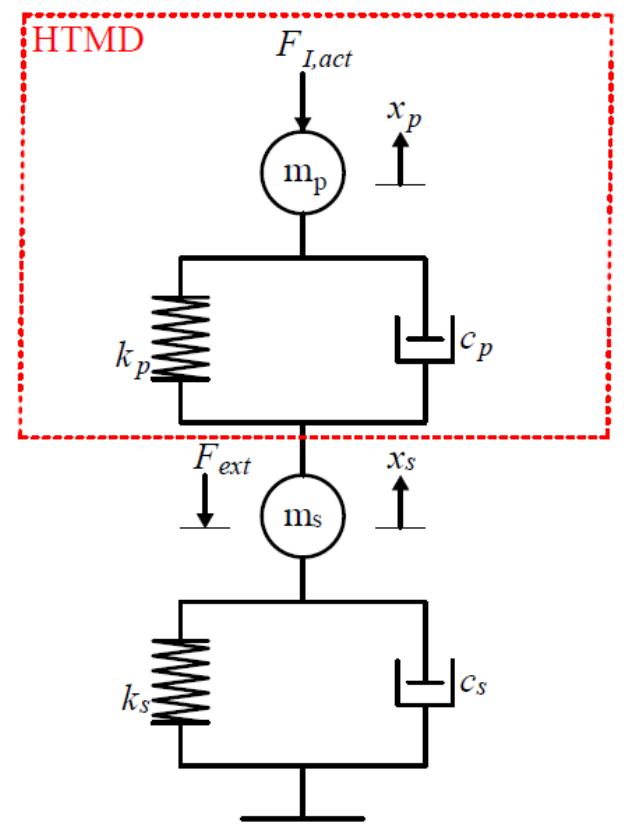

Figure 3-1. 2DOF model of the HTMD

The equations of the motion of the system are developed as:

$\left\{\begin{array}{c}m_{s} x_{s}(t)+\left(c_{s}+c_{p}\right) x_{s}(t)+\left(k_{s}+k_{p}\right) x_{s}(t)-c_{p} x_{p}(t)-k_{p} x_{p}(t)=F_{e x t}(t) \\ . \cdot m_{p} x_{p}(t)+c_{p} x_{p}(t)+k_{p} x_{p}(t)-c_{p} x_{s}(t)-k_{p} x_{s}(t)=F_{I, a c t}(t) \\ . .\end{array}\right.$

\subsection{Control algorithm}

Figure 3-2 shows the block diagram arrangement of the model. It is a closed loop system. As the sketch illustrates, both responses of the TMD mass and structure are continuously measured and used for feedback control. These two responses are measured as accelerations through the use of accelerometer sensors. Then, it is the purpose of the controller to calculate and generate the actuator control voltage to provide the inertia force to act on the TMD mass. 


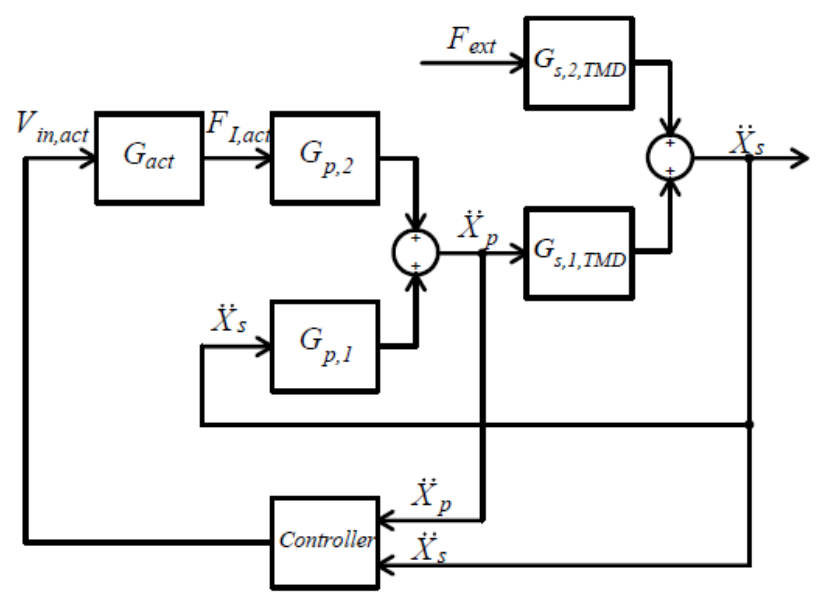

Figure 3-2. Block diagram arrangement of the structure with attached HTMD

Transferring ( 5 ) to the Laplace domain and rearranging it in terms of $X_{s}(s), X_{p}(s), F_{\text {ext }}(s)$ and $F_{I, a c t}(s)$ results in:

$\left\{\begin{array}{c}{\left[m_{s} s^{2}+\left(c_{s}+c_{p}\right) s+\left(k_{s}+k_{p}\right)\right] X_{s}(s)-\left[c_{p} s+k_{p}\right] X_{p}(s)=F_{\text {ext }}(s)} \\ {\left[m_{p} s^{2}+c_{p} s+k_{p}\right] X_{p}(s)-\left[c_{p} s+k_{p}\right] X_{s}(s)=F_{l, a c t}(s)}\end{array}\right.$

Introducing the following Transfer Functions:
a) $G_{s, 1, T M D}=\frac{X_{S}(s) s^{2}}{X_{p}(s) s^{2}}=\frac{-c_{p} s-k_{p}}{m_{s} s^{2}+\left(c_{s}+c_{p}\right) s+\left(k_{s}+k_{p}\right)}$
b) $G_{s, 2, T M D}=\frac{X_{s}(s) s^{2}}{F_{e x t}(s)}=\frac{s^{2}}{m_{s} s^{2}+\left(c_{s}+c_{p}\right) s+\left(k_{s}+k_{p}\right)}$
c) $G_{p, 1}=\frac{X_{p}(s) s^{2}}{X_{S}(s) s^{2}}=\frac{-c_{p} s-k_{p}}{m_{p} s^{2}+c_{p} s+k_{p}}$
d) $G_{p, 2}=\frac{X_{p}(s) s^{2}}{F_{I, a c t}(s)}=\frac{s^{2}}{m_{p} s^{2}+c_{p} s+k_{p}}$
e) $G_{a c t}=\frac{F_{I, a c t}(s)}{V_{\text {in, act }}(s)}=\frac{v_{a c t} s^{2}}{m_{a c t} s^{2}+c_{a c t} s+k_{a c t}} * \frac{1}{s+\varepsilon_{a c t}}$

Combining equations ( 6 ) and ( 7 ) leads to:

$\left\{\begin{array}{c}\ddot{x}_{s}=\ddot{x}_{p} G_{s, 1, T M D}+F_{e x t} G_{s, 2, T M D} \\ \ddot{x}_{p}=\ddot{x}_{s} G_{p, 1}+F_{I, a c t} G_{p, 2}\end{array}\right.$

Then the final transfer function of the system $\left(H_{H T M D}\right)$ between structural acceleration and external excitation force is given as

$\ddot{x}_{s}=\left(\ddot{x}_{s} G_{p, 1}+F_{I, a c t} G_{p, 2}\right) G_{s, 1, T M D}+F_{e x t} G_{s, 2, T M D} \therefore H_{H T M D}=\frac{\ddot{x}_{s}}{F_{e x t}}$

It should be noted that since $F_{I, a c t}$ depends on the responses of the structure and TMD, it is possible to simplify equation ( 9 ) by substituting $\ddot{x}_{s}$ and $\ddot{x}_{p}$ in $F_{I, a c t}$. As was shown in [13,18-24], the acceleration of the primary structure as the driving force and velocity of the TMD as the active damping force of the HTMD are two essential direct response feedback signals to improve the performance of the HTMD. Also, it was shown in [14] that the displacement and acceleration of the TMD are two feedback signals which can be used for adaptive tuning of the HTMD to deal with the problem of off-tuning of a passive TMD. 


\subsection{Gain optimization}

A system model is generated using the transfer functions introduced previously and by substituting the response of the structure and TMD in terms of $K_{i} X_{\text {Resp }}$, where $X_{\text {Resp }}$ is the response of the structure or TMD (i.e. displacement, velocity or acceleration) and $K_{i}$ is the feedback gain. It is then possible to introduce the feedback gains in Table 1 . The ranges of the individual gains were calculated using Root Locus (RL) analyses as was shown in [15].

Table 1. Control feedback gain ranges

\begin{tabular}{|c|c|}
\hline Type of response feedback & Gain Range \\
\hline$K_{3}$ Velocity of the main structure, $X_{\text {Resp }}=x_{s}$ & {$[-58,0]$} \\
\hline$K_{2}$ Acceleration of the main structure, $X_{\text {Resp }}=x_{s}$ & {$[-6.30,0]$} \\
\hline$K_{1}$ Displacement of the TMD, $X_{\text {Resp }}=x_{p}$ & {$[-1330,0]$} \\
\hline$K_{4}$ Velocity of the TMD, $X_{\text {Resp }}=x_{p}$ & {$[-100,0]$} \\
\hline$K_{5}$ Acceleration of the TMD, $X_{\text {Resp }}=x_{p}$ & {$[-0.835,0]$} \\
\hline
\end{tabular}

It is possible to use a Genetic Algorithm (GA) to optimize gains to achieve best performance of the HTMD. GA as a searching method has been employed to explore in the area of the feasible response and generate the optimized solution in that region. The aim of using HTMD is to minimize the response of the structure within a band of frequencies $\left(\omega_{i}\right)$ by using appropriate control gains. Hence, the critical values of the GA are the gains of the feedback system which should be optimized to reduce the FRF magnitude of the structure $\left(H_{H T M D}\left(\omega_{i}\right)\right)$ as the objective function $(\mathrm{OF})$. However, it should be noticed that the FRF of the controlled structure should be inside the boundary of the FRF of the uncontrolled structure in order to avoid higher responses for non-resonant frequencies. Hence, the FRF of the uncontrolled structure $H_{\text {unc }}\left(\omega_{i}\right)$ is considered as the constraint of the optimization problem. Summarizing these leads to:

OF : $\min \left(H_{H T M D}\left(\omega_{i}\right)\right), \quad \omega_{0}<\omega_{i}<\omega_{n}$

Constraint : S. T. $H_{\text {HTMD }}\left(\omega_{i}\right)<H_{\text {unc }}\left(\omega_{i}\right) \therefore H_{\text {HTMD }}\left(\omega_{i}\right)-H_{u n c}\left(\omega_{i}\right)<0$

$\omega_{0}<\omega_{i}<\omega_{n}$

This is a multi-objective nonlinear function with nonlinear semi-infinite constraints. To apply a GA to this problem, the Penalty Function method is introduced to create the Fitness Function of the GA given by

Fitness Function $=F_{i}+r_{p} \sum_{i=1}^{n} G_{i}$

where $r_{p}$ is the Penalty Factor, $G_{i}$ is the Constraint Function and $F_{i}$ is the Objective Function for $i$ th frequency. $n$ is the number of discreet frequencies. Implementing ( 11 ), both OF and Constraint Function convert to a single Fitness Function which can be applied to Matlab Optimization Toolbox [25], Multi-Objective Genetic Algorithm.

Table 2. GA parameters for HTMD's gain optimization

\begin{tabular}{|c|c|}
\hline Number of variables & 5 \\
\hline Population Size & 200 \\
\hline Selection Function & Tournament \\
\hline Tournament Size & 2 \\
\hline Reproduction/ Crossover fraction & 0.8 \\
\hline Mutation function & Adaptive feasible \\
\hline Crossover / Crossover fraction & Intermediate, ratio: 1.0 \\
\hline Migration Direction & 0.2 \\
\hline Migration fraction & 20 \\
\hline Migration Interval & 0.35 \\
\hline Pareto population fraction & Infinity \\
\hline Fitness Limit & 100 \\
\hline Stall generations & $10^{-5}$ \\
\hline Function tolerance & In serial \\
\hline Fitness function evaluation & \\
\hline
\end{tabular}


Using the properties in Table 2 for the GA, the optimized Critical Values (HTMD gains) are shown in Table 3. It should be noted that Upper Band and Lower Band of GA is very important since this is the area of choosing the critical values. In this case, these bands are set according to Table 1 . In addition, $r_{p}$, the Penalty Factor has been changed from $10^{1}$ to $10^{100}$. However, there is not a large alteration between the outputs of the optimization (HTMD gains), with maximum of $3 \%$ variation.

Table 3. Optimized gains to be used in HTMD to improve its performance

\begin{tabular}{|c|c|c|c|c|}
\hline$K_{3}$ & $K_{2}$ & $K_{1}$ & $K_{4}$ & $K_{5}$ \\
\hline 0 & -6.16 & -277.33 & -22.69 & -0.052 \\
\hline
\end{tabular}

Also, employing a similar method to deal with off-tuning as a result of the changing in the structural mass (which leads to variation of structural frequencies), it is possible to use similar Objective and Constraints Functions as equation ( 10 ) by having variable structural mass such as $m_{s, i}$ instead of a fixed structural mass $m_{s}$. The result of the optimization is shown in Table 4.

Table 4. Optimized gains to be used in HTMD to deal with off-tuning

\begin{tabular}{|c|c|c|c|c|}
\hline$K_{3}$ & $K_{2}$ & $K_{1}$ & $K_{4}$ & $K_{5}$ \\
\hline 0 & -6.25 & -396.80 & -30.74 & -0.09 \\
\hline
\end{tabular}

These results are used in both the computer analyses and experimental investigation detailed in the next section.

\subsection{Stability check}

The stability of a SIMO system can be investigated by two techniques. Firstly, by evaluating the zeros of the closed loop system (i.e. roots of $1+K_{c}(s) G(s)$ where $K_{c}(s) G(s)$ is the transfer function of the loop in Figure 3-3). Secondly, by using Nyquist plot of $K_{c}(s) G(s)$ and determining whether it does not encircle point $(-1,0)[26,27]$.

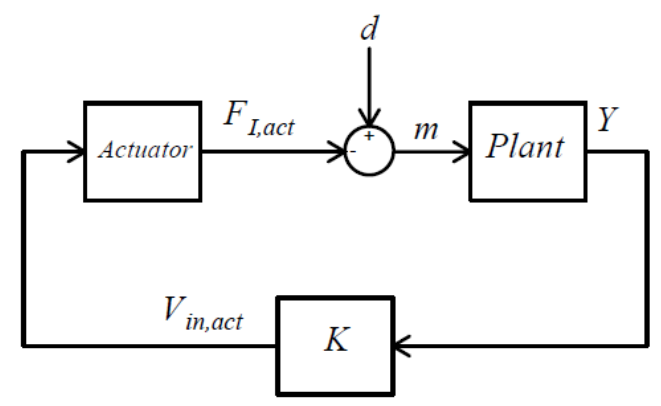

Figure 3-3. Block diagram of typical feedback control scheme

Developing the closed loop transfer function from Figure 3-3 leads to:

a) $m=d-F_{I, a c t} \therefore m=d-\{k\}\{Y\} G_{\text {act }}$

b) $\{Y\}=m\left\{G_{\text {Plant }}\right\}$

Substituting two parts of the equation results in

$$
\frac{d}{m}=\{k\}\left\{G_{\text {Plant }}\right\} G_{\text {act }}
$$


where $\{k\}$ is the feedback vector of the different types of outputs (i.e. structure or TMD's responses) and $\left\{G_{\text {Plant }}\right\}$ is the vector of the transfer functions between the structure or TMD responses and the control force.

For the first technique, the zeros of the following equation should be on the left hand side of the $s$-plane.

$$
1+\{k\}\left\{G_{\text {Plant }}\right\} G_{\text {act }}
$$

Also, for the second technique, the Nyquist plot of the following equation should not encircle point $(-1,0)$.

$$
\{k\}\left\{G_{\text {Plant }}\right\} G_{\text {act }}
$$

As Figure 3-4 demonstrates, all poles and zeros of the closed loop system are on the left hand side of the $s$-plane, which indicates a stable system. This is the HTMD with the gains given in Table 3.

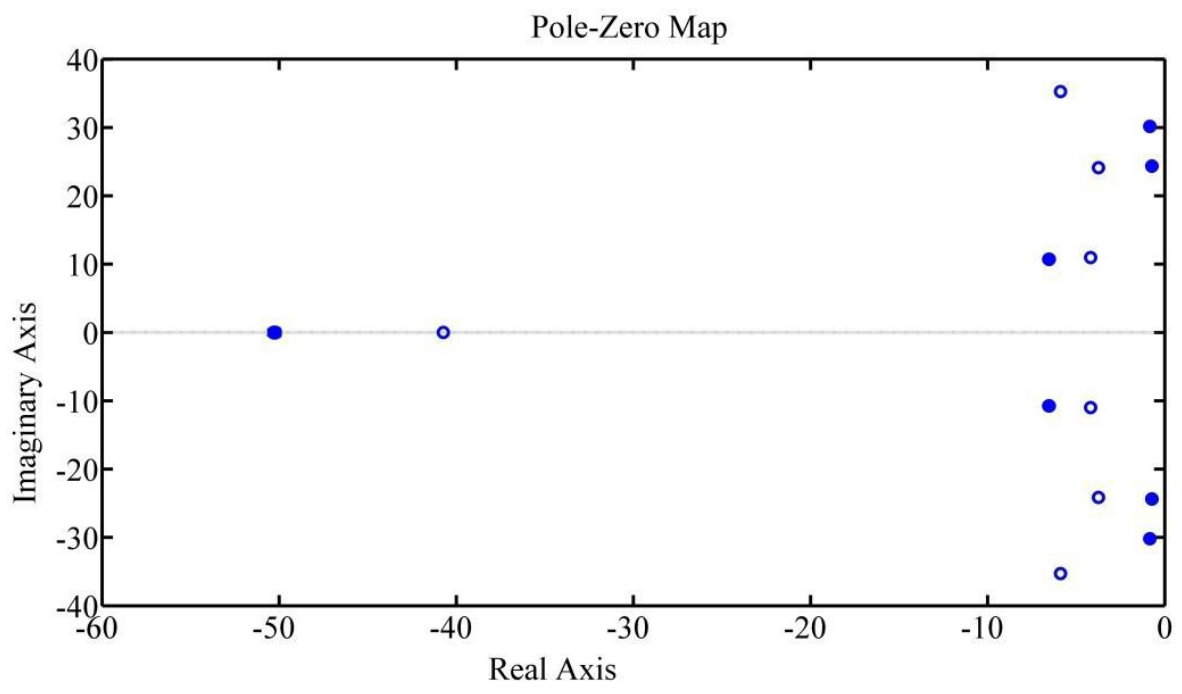

Figure 3-4. Pole-Zero map of the closed loop HTMD system

Also, Figure 3-5 illustrates the Nyquist plot of the same system. Clearly, there is no encirclement around the point (-1, 0 ), which indicates stability of the closed loop system. Again, this is the HTMD with gains as given in Table 3 . The same methodology can be used to check the stability of the system with the gains in Table 4. In both cases the conditions for stability were met.

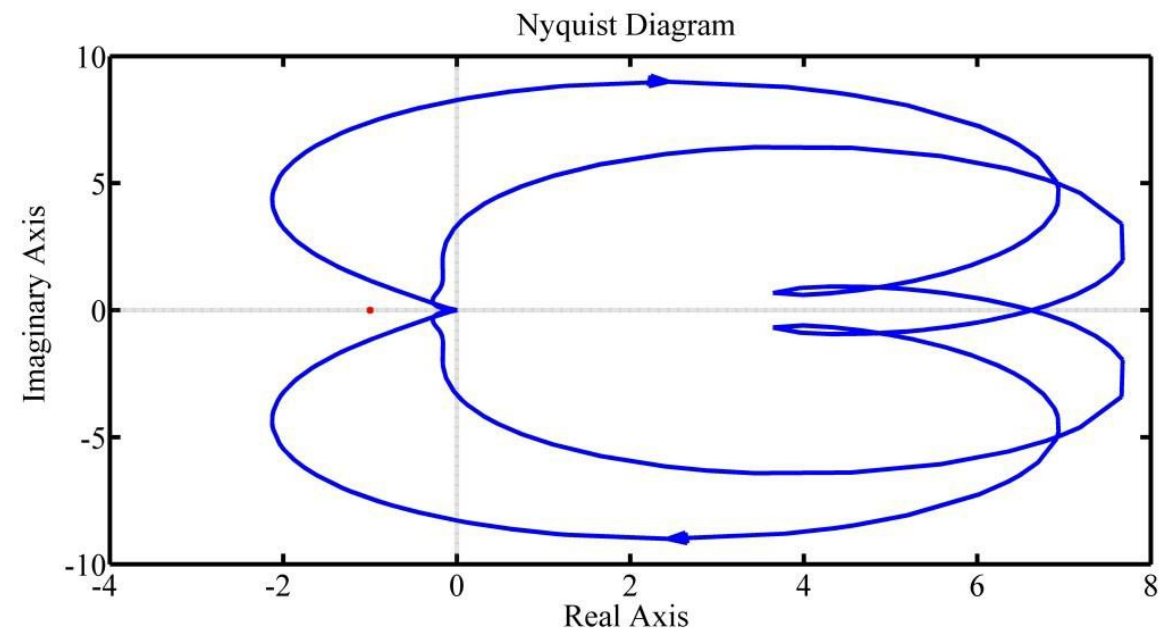

Figure 3-5. Nyquist plot of the closed loop HTMD system 


\section{EXPERIMENTAL RESULTS}

To perform the test, one accelerometer was placed at the center of the slab and a second accelerometer on the TMD mass. These accelerometers were Endevco Model 7754A-1000. Also, a dSpace control unit (model ACE1103 consisting of a DS1103 PowerPC GX/1 GHz controller board and CLP1103 LED panel) was employed to apply the control algorithms to the system. The accelerations of the TMD mass and structure were the two input signals to the controller. Also three identical band pass filters were applied to the model before each integral to remove frequencies below $0.7 \mathrm{~Hz}$ and above $50 \mathrm{~Hz}$.

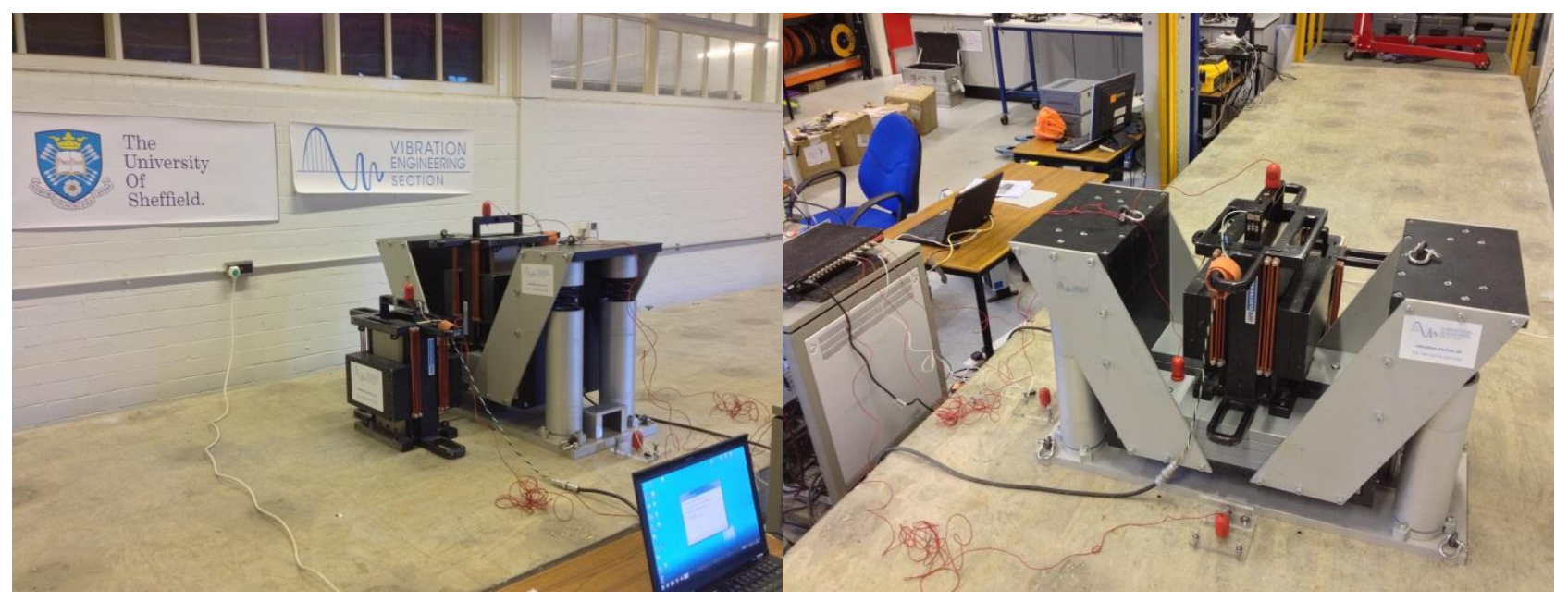

Figure 4-1. HTMD on the laboratory slab strip

\subsection{HTMD performance test}

Figure 4-2 shows the result of both analytical simulations and experimental tests using the gains in Table 3 . This is the FRF magnitude between the external excitation and structural acceleration for the uncontrolled structure, structure with passive TMD and structure with HTMD. This figure both verifies the analytical model proposed and shows that the response of the structure is reduced further using the HTMD compared to the passive TMD.

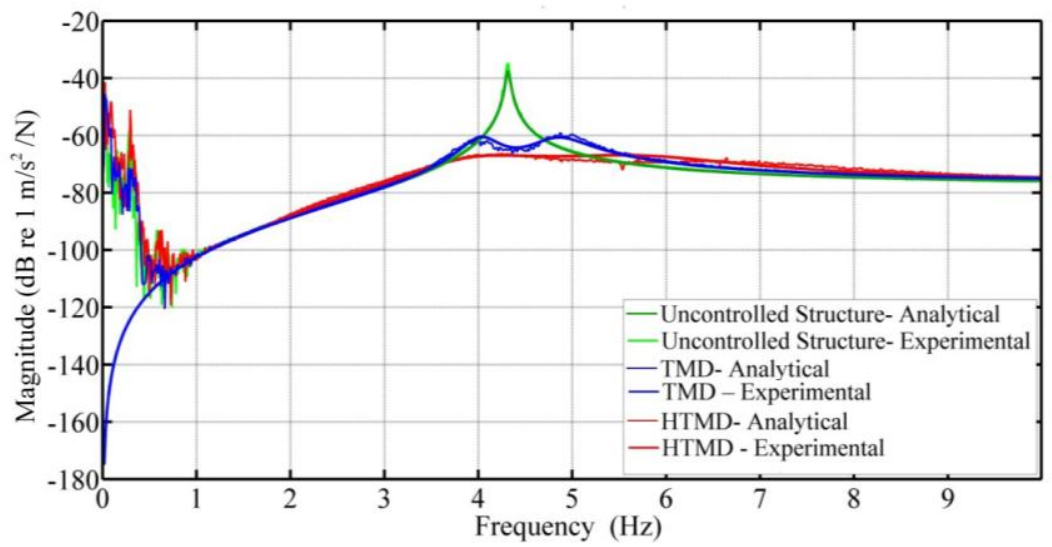

Figure 4-2. FRF magnitude comparison of the uncontrolled structure, structure with TMD and structure with HTMD from both analytical and experimental results 
Table 5 compares the numerical results of the measurements. It shows that the HTMD has $52 \%$ and $30 \%$ reduction in the maximum magnitude of the FRF and magnitude of FRF at the resonant frequency, respectively, in comparison with the passive TMD.

Table 5. Response reduction comparison between uncontrolled structure, structure with TMD and HTMD

\begin{tabular}{|c|c|c|c|}
\hline & $\begin{array}{c}\text { Uncontrolled } \\
\text { Structure }\end{array}$ & $\begin{array}{c}\text { Structure with } \\
\text { TMD }\end{array}$ & $\begin{array}{c}\text { Structure with } \\
\text { HTMD }\end{array}$ \\
\hline Max. response magnitude $\left(\left(\mathrm{m} / \mathrm{s}^{2}\right) / \mathrm{N}\right)$ & 0.0136 & 0.000942 & 0.000449 \\
\hline Reduction of the max. response & N/A & $93 \%$ & $97 \%$ \\
\hline Response magnitude on resonance $\left(\left(\mathrm{m} / \mathrm{s}^{2}\right) / \mathrm{N}\right)$ & 0.0136 & 0.000639 & 0.000447 \\
\hline Reduction of response in resonance & N/A & $95 \%$ & $97 \%$ \\
\hline
\end{tabular}

\subsection{Off-tuning test}

This is the experiment where the result of the gains in Table 4 is employed to deal with off-tuning. To implement the offtuning to the system, since it is not convenient to change the mass of structure, the TMD passive mass was changed by adding or removing masses on it (Figure 4-3). This resulted in an out-of-tune TMD. The performance of the HTMD at a range of frequencies is compared with that of the passive TMD.

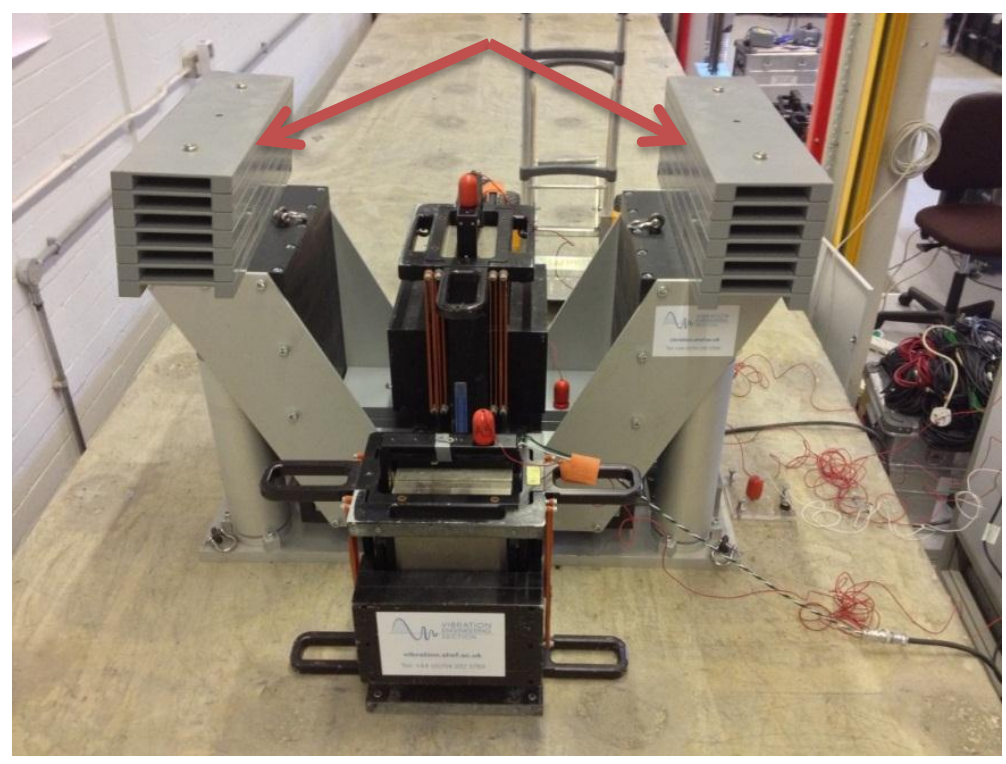

Figure 4-3. Masses added to the HTMD for reducing its resonant frequency

Figure 4-4, Figure 4-5 and Figure 4-6 show the structural FRF magnitude comparison between the HTMD and passive TMD using different masses (i.e. frequencies) on the TMD/HTMD. These are the FRFs between external excitation force and the resulting structural acceleration. 


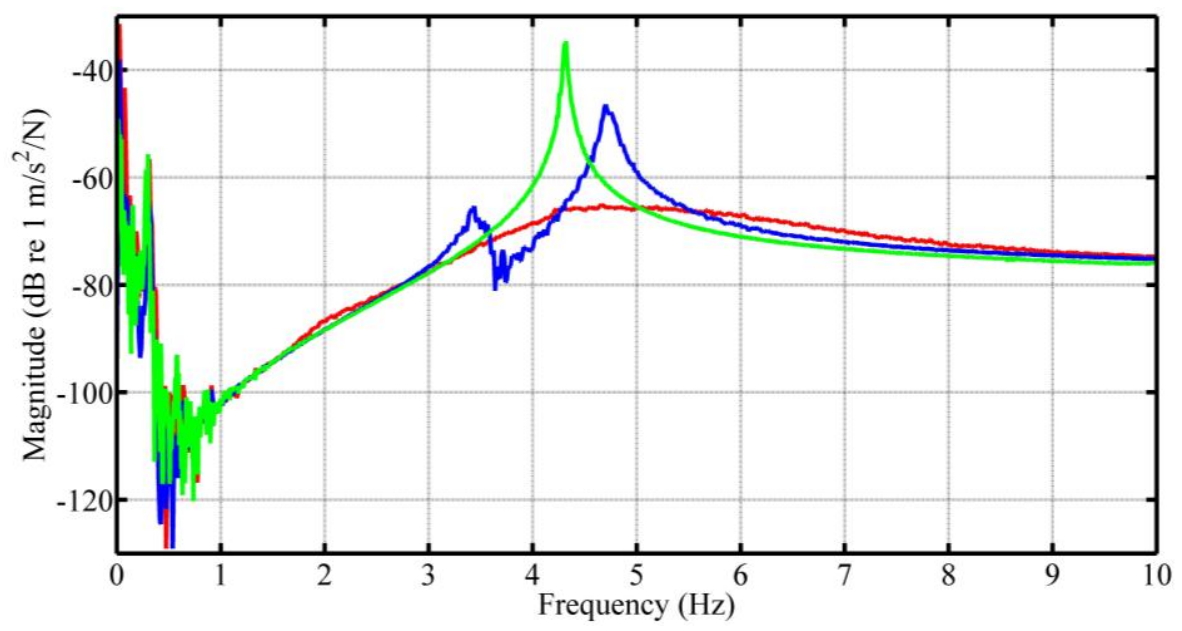

Figure 4-4. FRF comparison of structure with TMD and HTMD, $m_{p}=500 \mathrm{~kg}$; uncontrolled structure (green), structure with TMD (blue), structure with HTMD (red)

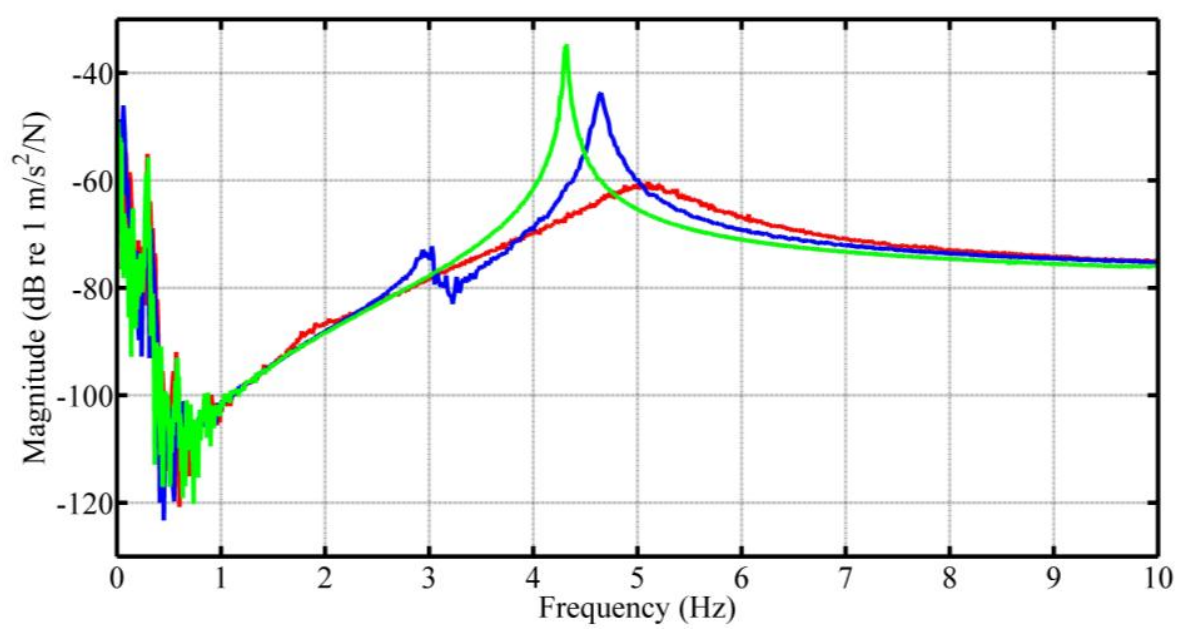

Figure 4-5. FRF comparison of structure with TMD and HTMD, $m_{p}=700 \mathrm{~kg}$; uncontrolled structure (green), structure with TMD (blue), structure with HTMD (red) 


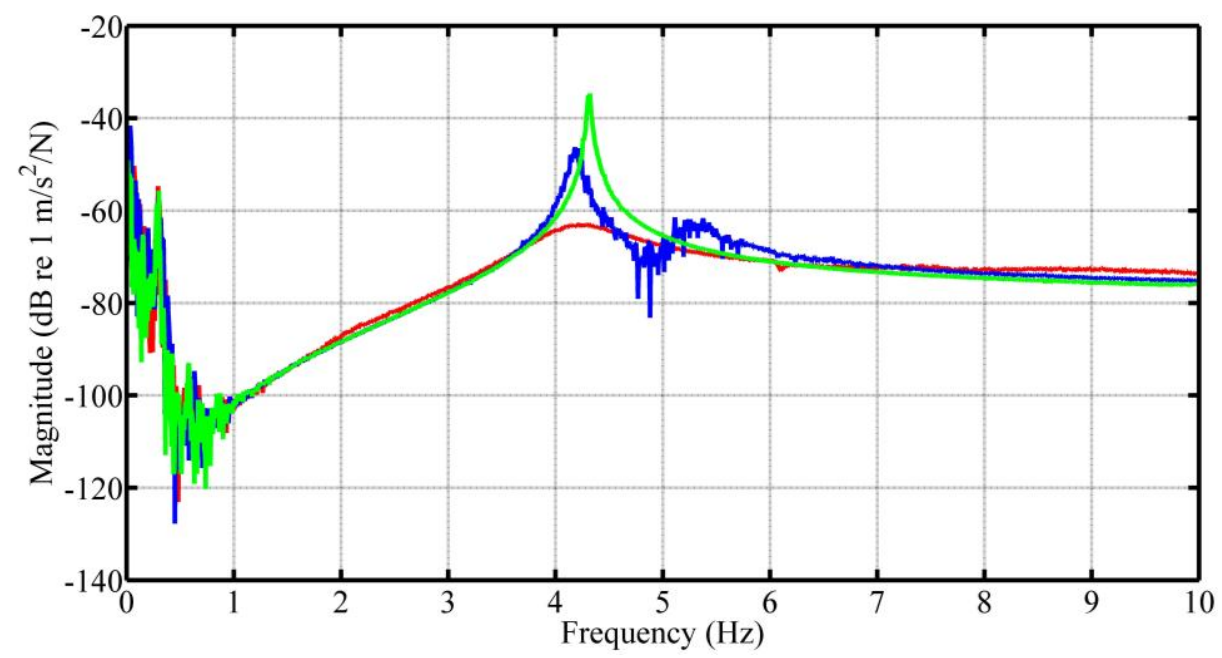

Figure 4-6. FRF comparison of structure with TMD and HTMD, $m_{p}=250 \mathrm{~kg}$; uncontrolled structure (green), structure with TMD (blue), structure with HTMD (red)

Table 6 shows the results of the experiments. It shows that the HTMD has an appropriate performance in the presence of off-tuning whereas the passive TMD is much less effective.

Table 6. Comparison of the FRF magnitude with different frequencies of the TMD and HTMD

\begin{tabular}{|c|c|c|c|}
\hline & Mass on TMD & Max. response magnitude $\left(\left(\mathrm{m} / \mathrm{s}^{2}\right) / \mathrm{N}\right)$ & Reduction of max. response \\
\hline TMD & \multirow{2}{*}{500} & 0.004772 & N/A \\
\hline HTMD & & 0.0005455 & $89 \%$ \\
\hline TMD & \multirow{2}{*}{700} & 0.006545 & N/A \\
\hline HTMD & & 0.0009721 & $85 \%$ \\
\hline TMD & \multirow{2}{*}{250} & 0.004857 & N/A \\
\hline HTMD & & 0.00070771 & $85 \%$ \\
\hline
\end{tabular}

\section{CONCLUSIONS}

Herein, the authors have analytically and experimentally investigated the HTMD proposed in [14] and [15]. This is the performance of the Hybrid Tuned Mass Damper in comparison with passive TMD to reduce the structural response in addition to dealing with off-tuning issues.

Also, the authors have proposed a new optimization method for HTMDs using a Genetic Algorithm to generate the appropriate control gains. The results verify the proposed analytical model and illustrate that the performance of the HTMD is more enhanced in comparison with a passive TMD and is less susceptive to off-tuning.

\section{ACKNOWLEDGMENTS}

The authors would like to acknowledge the financial assistance provided by the UK Engineering and Physical Sciences Research Council (EPSRC) through Leadership Fellowship grant "Advanced Technologies for Mitigation of HumanInduced Vibration" (Ref. EP/J004081/1) and platform grant "Dynamic Performance of Large Civil Engineering Structures: An Integrated Approach to Management, Design and Assessment” (Ref. EP/G061130/1). 


\section{REFERENCES}

[1] Institution of Structural Engineers, Dynamic Performance Requirements for Permanent Grandstands Subject to Crowd Action: Recommendations for Management, Design and Assessment, in Institution of Structural Engineers SD/03/26(November), pp. 1-22, The Institution of Structural Engineers, The Department for Communities and Local Government, The Department for Culture Media and Sport, London (2008).

[2] M. Kasperski and H. Niemann, "Man induced vibrations of a stand structure," Proc., EURODYN, 977-983 (1993).

[3] J. G. Parkhouse and D. J. Ewins, "Crowd-induced rhythmic loading," Structures \& Buildings 159(SB5), 247259 (2006).

[4] B. R. B. Ellis, T. Ji, J. D. J. D. Littler, and E. E. T. Al, "The response of grandstands to dynamic crowd loads," Proceedings of the Institution of Civil Engineers: Structures and Buildings 140(4), 355-365 (2000) [doi:10.1680/stbu.2000.140.4.355].

[5] G. W. Housner, L. A. Bergman, T. K. Caughey, A. G. Chassiakos, R. O. Claus, S. F. Masri, R. E. Skelton, T. T. Soong, B. F. Spencer, et al., "Structural Control: Past, Present, and Future," Journal of Engineering Mechanics 123(9), 897 (1997) [doi:10.1061/(ASCE)0733-9399(1997)123:9(897)].

[6] D. S. Nyawako and P. Reynolds, "Technologies for mitigation of human-induced vibrations in civil engineering structures," The Shock and Vibration Digest 39(6), 465-493, Sage (2007) [doi:10.1177/0583102407084286].

[7] T. Soong and J. B. F. Spencer, "Supplemental energy dissipation: state-of-the-art and state-of-the-practice," Engineering Structures 24(3), 243-259 (2002) [doi:10.1016/S0141-0296(01)00092-X].

[8] N. D. Sims, "Vibration absorbers for chatter suppression: A new analytical tuning methodology," Journal of Sound and Vibration 301(3-5), 592-607 (2007) [doi:10.1016/j.jsv.2006.10.020].

[9] J.-S. Bae, J.-H. Hwang, J.-H. Roh, J.-H. Kim, M.-S. Yi, and J. H. Lim, "Vibration suppression of a cantilever beam using magnetically tuned-mass-damper," Journal of Sound and Vibration 331(26), 5669-5684, Elsevier (2012) [doi:10.1016/j.jsv.2012.07.020].

[10] G. Aguirre, M. Gorostiaga, T. Porchez, and J. Muñoa, "Self-tuning semi-active tuned-mass damper for machine tool chatter suppression," in ISMA2012-USD2012, pp. 109-124 (2012).

[11] P. Reynolds and A. Pavic, "The dynamic performance of sports stadia under crowd dynamic loading at concert events," Sixth European Conference on Dynamics EURODYN, Paris, Fra, 473-479, Millpress, Rotterdam (2005).

[12] A. Pavic and P. Reynolds, "Experimental verification of novel 3DOF model for grandstand crowdstructure dynamic interaction," 26th International Modal Analysis ... 6399 (2008).

[13] C. M. Wang, N. Yan, and T. Balendra, "Control on Dynamic Structural Response Using ActivePassive Composite-Tuned Mass Dampers," Journal of Vibration and Control 5(3), 475-489 (1999) [doi:10.1177/107754639900500308].

[14] N. Noormohammadi and P. Reynolds, "Employing hybrid tuned mass damper to solve off-tuning problems for controlling human induced vibration in stadia," in IMAC XXXI, Orange County, California, USA (2013).

[15] N. Noormohammadi and P. Reynolds, "Control of human induced vibrations in stadia using a hybrid tuned mass damper," in Noise and Vibration Engineering, ISMA2012, pp. 1119-1132 (2012).

[16] D. Nyawako and P. Reynolds, "Response-dependent velocity feedback control for mitigation of human-induced floor vibrations," Smart Materials and Structural 18(7), 1-14, 2009 IOP Publishing Ltd, UK (2009) [doi:10.1088/0964-1726/18/7/075002].

[17] P. Reynolds, "The Effects of Raised Access Flooring on the Vibrational Performance of Long-Span Concrete Floors," p. -, University of Sheffield (2000).

[18] I. Nishimura, T. Kobori, and M. Sakamoto, "Active passive composite tuned mass damper," PROCEEDINGS OF THE SEMINAR ON SEISMIC ISOLATION, 737-748 (1993).

[19] S. Ohrui, T. Kobori, S. Mitsuta, N. Koshika, I. Nishimura, K. Sasaki, A. Kondo, I. Fukushima, and M. Sakamoto, "An Active-Passive Composite Tuned Mass Damper for Vibration Control of A Building," in Second International Conference on Motion and Vibration Control, pp. 569-574, Yokohama, Japan (1994).

[20] I. Nishimura, T. Kobori, M. Sakamoto, N. Koshika, K. Sasaki, S. Ohrui, H. Search, C. Journals, A. Contact, et al., "Active tuned mass damper," Smart Materials and 1(4), 306-311, IOP Publishing (1992) [doi:10.1088/0964-1726/1/4/005]. 
[21] I. Nishimura, T. Yamada, M. Sakamoto, and T. Kobori, "Control performance of active-passive composite tuned mass damper," Smart Materials and Structures 7(5), 637-653, IOP Publishing (1998) [doi:10.1088/0964-1726/7/5/008].

[22] I. Nishimura, M. Sakamoto, T. Yamada, N. Koshika, and T. Kobori, "Acceleration feedback method applied to active-passive composite tuned mass damper," Journal of Structural Control 1(1-2), 103-116, Wiley Online Library (1994).

[23] I. Fukushima, K. Sasaki, I. Nishimura, N. Koshika, M. Sakamoto, and T. Kobori, "Development and application of active-passive composite tuned mass damper to high-rise building," Proc. of the Pacific ... (1995).

[24] K. R. Crescent, N. Yang, C. Wang, and T. Balendra, "Composite mass dampers for vibration control of wind-excited towers," Journal of sound and vibration 213(2), 301-316 (1998).

[25] Matlab, Matlab Optimization Toolbox User's Guide (2012).

[26] R. C. Dorf and R. H. Bishop, "Modern Control Systems," p. -831, Prentice Hall, New Jersey (2001).

[27] D. S. Nyawako and P. Reynolds, "LQR controller for an in-service floor," Dynamics of Civil Structures, Volume 4, 227-237, Springer (2011) [doi:10.1007/978-1-4419-9831-6_24]. 